\title{
Hypoxia-Induced Neonatal Seizures Diminish Silent Synapses and Long-Term Potentiation in Hippocampal CA1 Neurons
}

\author{
Chengwen Zhou, ${ }^{1,2 \star}$ Jocelyn J. Lippman Bell, ${ }^{1,2 \star}$ Hongyu Sun, ${ }^{1,2 \star}$ and Frances E. Jensen ${ }^{1,2,3}$ \\ ${ }^{1}$ Department of Neurology, Division of Neuroscience, Children's Hospital, Boston Massachusetts 02115, ${ }^{2}$ Harvard Medical School, Boston Massachusetts \\ 02115, and 'Program in Neurobiology, Harvard Medical School, Boston Massachusetts 02115
}

\begin{abstract}
Neonatal seizures can lead to epilepsy and long-term cognitive deficits into adulthood. Using a rodent model of the most common form of human neonatal seizures, hypoxia-induced seizures (HS), we aimed to determine whether these seizures modify long-term potentiation (LTP) and silent NMDAR-only synapses in hippocampal CA1. At 48-72 h after HS, electrophysiology and immunofluorescent confocal microscopy revealed a significant decrease in the incidence of silent synapses, and an increase in AMPARs at the synapses. Coincident with this decrease in silent synapses, there was an attenuation of LTP elicited by either tetanic stimulation of Schaffer collaterals or a pairing protocol, and persistent attenuation of LTP in slices removed in later adulthood after P10 HS. Furthermore, postseizure treatment in vivo with the AMPAR antagonist 2,3-dihydroxy-6-nitro-7-sulfonyl-benzo[f]quinoxaline (NBQX) protected against the HS-induced depletion of silent synapses and preserved LTP. Thus, this study demonstrates a novel mechanism by which early life seizures could impair synaptic plasticity, suggesting a potential target for therapeutic strategies to prevent long-term cognitive deficits.
\end{abstract}

\section{Introduction}

The incidence of seizures is high in the neonatal period, with the most common cause being hypoxic encephalopathy. These seizures can result in later-life epilepsy (Silverstein and Jensen, 2007) and be associated with learning disabilities and cognitive disorders such as autism (Ronen et al., 2007; Berg, 2011). Neonatal seizures can be resistant to conventional antiepileptic drugs, indicating that underlying mechanisms may differ from those in adulthood (Rakhade and Jensen, 2009). Because these seizures occur during a critical period of synaptic development (Huttenlocher and Wollmann, 1991; Jensen et al., 1992; Holmes and Ben-Ari, 2007; Rakhade and Jensen, 2009; Scantlebury et al., 2010), we hypothesized that early life hypoxia-induced seizures alter normal patterning of synaptic development and plasticity important for learning, such as long-term potentiation (LTP).

In rats, brief hypoxia-induced seizures (HS) at postnatal day (P) 10 result in a high incidence of spontaneous seizures (Rakhade et al., 2011) and cognitive deficits (Yang et al., 2004; Mikati et al., 2005) later in life. Also, early life HS cause rapid posttranslational

\footnotetext{
Received Sept. 22, 2011; accepted 0ct. 11, 2011.

Author contributions: C.Z., J.J.L.B., H.S., and F.E.J. designed research; C.Z., J.J.L.B., and H.S. performed research; C.Z., J.J.L.B., H.S., and F.E.J. analyzed data; C.Z., J.J.L.B., H.S., and F.E.J. wrote the paper.

This work was supported by funding from National Institutes of Health NS 31718 (F.E.J.), DP1 0 D003347 (F.E.J.), NICHD 5T32HD007466 (C.Z.), and NINDS NRSA F32NS068161-02 (J.L.B.) and by core support from the Intellectual and Developmental Disorders Research Center Grant (NIH NICHHD P30 HD18655). We thank Peter Klein for technical assistance and Nikolaus Sucher, PhD, for his helpful comments on this manuscript.

${ }^{*}$ C.Z., J.J.L.B., and H.S. contributed equally to this work.

Correspondence should be addressed to Frances Jensen, 300 Longwood Avenue, CLS14073, Boston MA 02115. E-mail: frances.jensen@childrens.harvard.edu.

DOI:10.1523/JNEUROSCI.4838-11.2011

Copyright $\odot 2011$ the authors $\quad 0270-6474 / 11 / 3118211-12 \$ 15.00 / 0$
}

modifications of AMPARs in hippocampal area CA1, with transient and early increases in GluA1 subunit phosphorylation at Ser831 and Ser845 (Rakhade et al., 2008), sites associated with subunit trafficking and enhancement of AMPAR currents (Liao et al., 2001). Consistently, AMPAR spontaneous and miniature EPSC amplitude and frequency in CA1 pyramidal neurons increase within 1-3 h after HS in P10 rats (Rakhade et al., 2008).

During this period, many immature synapses contain NMDARs but not AMPARs, remaining silent due to $\mathrm{Mg}^{2+}$ blockade of NMDARs at resting membrane potentials (Isaac et al., 1995; Liao et al., 1995; Busetto et al., 2008). Over development, synapses become functional by activity-dependent insertion of AMPARs (Petralia et al., 1999; Malinow and Malenka, 2002; Kerchner and Nicoll, 2008). As a result, mature glutamatergic synapses coexpress AMPARS with NMDARs, where AMPARmediated membrane depolarization removes the $\mathrm{Mg}^{2+}$ blockade, allowing for NMDAR activation. Silent synapse conversion has also been demonstrated during synaptic strengthening that occurs during LTP (Marie et al., 2005; Lisman and Raghavachari, 2006; Thiagarajan et al., 2007; Kerchner and Nicoll, 2008).

The present study examined silent synapse expression and synaptic plasticity in hippocampal CA1 in slices $48-72 \mathrm{~h}$ after in vivo $\mathrm{HS}$ in $\mathrm{P} 10$ rats. We chose this time point because it follows the peak in posttranslational GluA1 changes (12-24 h after seizure) (Rakhade et al., 2008). Also, AMPAR antagonist treatment given within $48 \mathrm{~h}$ after HS can prevent these GluA1 alterations and reverse long-term changes in seizure susceptibility (Koh et al., 2004). We show significant loss of silent synapses at $48-72 \mathrm{~h}$ after HS compared with controls, and this was coincident with a markedly reduced capacity for LTP, which persisted into adulthood. Furthermore, postseizure 
treatment in vivo with AMPAR antagonist 2,3-dihydroxy-6-nitro-7sulfonyl-benzo[f]quinoxaline (NBQX), at doses we have previously shown block post-HS increases in GluA1 Ser831 and Ser845 phosphorylation, prevented the silent synapses loss and LTP impairment. Because silent synapses are critical to LTP induction (Kerchner and Nicoll, 2008), these data suggest that changes in silent synapses and AMPAR composition may contribute to impaired network plasticity and LTP, and may be relevant to understanding the mechanism whereby neonatal seizures lead to cognitive deficits.

\section{Materials and Methods}

Animals. Litters of male Long-Evans hooded rats (Charles River Laboratories, 10 pups per litter) were subjected to HS at P10, as described previously (Jensen et al., 1998). Age- and weight-matched littermates not exposed to hypoxia served as normoxic controls. Hypoxia-induced tonic-clonic seizures, automatisms followed by head and limb movements and myoclonic jerks, were observed. Only animals that had $>5$ tonic-clonic seizures were included in the study (93\%). In our model, seizures occur in $>95 \%$ of the P10 rats exposed to hypoxia, so only a small number of animals could be used to determine whether hypoxia alone or seizure activity influence the silent synapses and synaptic plasticity.

All rat pups were returned to their dams within an hour after the experiment. All procedures were approved by and in accordance with the guidelines of the Animal Care and Use Committee at Children's Hospital Boston (Boston, MA) and the National Institutes of Health Guide for the Care and Use of Laboratory Animals. All efforts were made to minimize animal suffering and the number of animals used.

Hippocampal slice preparation. Hippocampal slices were prepared as described in detail previously (Sanchez et al., 2005). Rat pups were decapitated at intervals up to $72 \mathrm{~h}$ following HS, as were adults. Agematched littermate rats not exposed to hypoxia were used as controls. Brains were rapidly dissected from the skull and placed for sectioning in ice-cooled cutting solution containing the following (in $\mathrm{mm}$ ): $210 \mathrm{su}-$ crose, $2.5 \mathrm{KCl}, 1.02 \mathrm{NaH}_{2} \mathrm{PO}_{4}, 0.5 \mathrm{CaCl}_{2}, 10 \mathrm{MgSO}_{4}, 26.19 \mathrm{NaHCO}_{3}$, and 10 D-glucose, $\mathrm{pH} 7.4$, bubbled with $95 \% \mathrm{O}_{2} / 5 \% \mathrm{CO}_{2}$ at $4^{\circ} \mathrm{C}$. Coronal hippocampal slices (300 $\mu \mathrm{m}$ thickness) were sectioned from the middle third of hippocampus with a vibratome (Leica VT1000S) in cutting solution. Slices were incubated in oxygenated artificial CSF (ACSF), containing the following (in mM): $124 \mathrm{NaCl}, 5 \mathrm{KCl}, 1.25 \mathrm{NaH}_{2} \mathrm{PO}_{4}, 2 \mathrm{CaCl}_{2}$, $1.3 \mathrm{MgSO}_{4}, 26 \mathrm{NaHCO}_{3}$, and 10 D-glucose, pH 7.4 (Sanchez et al., 2005) and transferred to $32^{\circ} \mathrm{C}$ for $30 \mathrm{~min}$. Then, slices were kept at room temperature for at least $1 \mathrm{~h}$ before electrophysiological recordings.

Triple immunostaining analysis of silent synapses in hippocampus. Staining and analysis were based on, but heavily modified from, previous methods (Mokin and Keifer, 2006). Forty-eight hours following HS at $\mathrm{P} 10$, rats and age-matched littermate controls were anesthetized with 100 $\mathrm{mg} / \mathrm{kg}$ pentobarbital and transcardially perfused with PBS, followed by $4 \%$ paraformaldehyde. Brains were dissected out and postfixed for $1 \mathrm{~h}$, then cryoprotected in $30 \%$ sucrose until saturated. Brains were sectioned to $20 \mu \mathrm{m}$ thickness using a cryostat (Leica). After washing sections in PBS, we used antigen retrieval by microwave irradiation in $0.2 \%$ sodium citrate buffer, $\mathrm{pH}$ 6.0. Sections were then washed and incubated for $1-2 \mathrm{~h}$ at room temperature in a $10 \%$ normal goat serum blocking solution, then overnight in a moisture chamber at $4^{\circ} \mathrm{C}$ in mouse anti-NR1 $(1: 125$, AbCam). After washing with PBS, sections were then incubated in Alexa488 secondary for $1 \mathrm{~h}$, washed with PBS, and then primary and secondary antibody incubation was repeated for rabbit anti-GluA1 (1:6000, ThermoScientific) followed with Alexa-594 secondary and sheep antisynaptophysin (1:500, AbCam) followed with Alexa-647 secondary. (All secondary antibodies were from Invitrogen, used at 1:1000.).

Images were acquired on a Zeiss LSM510 confocal microscope with a $63 \times$ oil-immersion objective $(\mathrm{NA}=1.4)$. Slides were blinded, then images were taken from stratum radiatum (s. radiatum) in CA1, $\sim 100 \mu \mathrm{m}$ from the pyramidal neuron somas, in the middle third of the hippocampus (see Fig. $4 A, B$ ). Using a digital zoom of 3 and line averaging of 2, $10-12 \mu \mathrm{m}$ stacks $(0.25 \mu \mathrm{m} z$-intervals, 12-bit) were scanned. Images were analyzed blind using MetaMorph software. To analyze percentage of NR1-only synapses, a $240 \times 140$ pixel field was chosen in a single plane in a semirandom manner (chosen to not include areas that contained somas, due to an altered distribution of the three markers on somas vs in the neuropil). The stack was reduced to the plane containing the box, the plane below, and the plane above, and run through a low-pass filter to reduce noise. Channels were separated and thresholded, with levels determined by measuring the intensity of seven distinct areas near puncta. To avoid under-thresholding, the lowest value was dropped and the remaining six values were averaged to give a minimum threshold level at which puncta could be counted. Threshold was at least two times the background intensity (Crump et al., 2001). Puncta were defined by distinct intensity peaks above threshold, based on line scans through adjacent puncta. Puncta were counted in one plane in the green channel (NR1), then the puncta ROIs were transferred to the extended stack (three planes) of the blue channel (synaptophysin). ROIs not in contact with blue puncta were deleted, as our criteria for calling NR1 synaptic was contact with synaptophysin puncta in at least one plane. The remaining ROI puncta were transferred to an extended stack of the GluA1 channel to determine how many of the NR1/synaptophysin puncta were also in contact with GluA1 puncta. From this, we calculated the percentage of NR1/synaptophysin synapses that did not also contain GluA1 (NR1-only silent synapses). GluA1 puncta that were not in contact with NR1 puncta were not included in this analysis. To ensure that our results were not an artifact of the chosen threshold, a subset of images were analyzed at 5, 10, $15,20,25$, and $30 \%$ of the maximum threshold of the red channel (GluA1), as described previously (Nie et al., 2010). To measure the number of GluA1/synaptophysin overlapping puncta, we conducted a similar analysis in a subset of the images analyzed for silent synapses, but counted GluA1, instead of NR1, puncta that were in contact with synaptophysin.

Electrophysiology. Whole-cell patch-clamp recordings were made from hippocampal CA1 pyramidal neurons in brain slices using infrareddifferential interference contrast microscope as described previously (Rakhade et al., 2008). Both normoxic control and post-HS CA1 pyramidal neurons showed similar appearances without any edema symptoms in slices (Sanchez et al., 2001; Rakhade et al., 2011). The patch-pipette internal solution contained the following (in $\mathrm{mM}$ ): 110 Cs-methanesulfonate, 10 TEA-Cl, $4 \mathrm{NaCl}, 2 \mathrm{MgCl}_{2}, 10$ EGTA, 10 HEPES, 4 ATP-Mg, and 0.3 GTP, pH 7.25, QX-314 (5 mM), phosphocreatine (7 $\mathrm{mM})$, and creatine phosphokinase (17 unit/ml). Filled electrodes had resistances of 2-5 M $\Omega$. EPSCs were elicited at 10 s intervals. Minimally evoked EPSCs were recorded as described previously (Isaac et al., 1996; Rakhade et al., 2008). GABA receptors were blocked with picrotoxin (60 $\mu \mathrm{M})$ for all recordings unless otherwise specified. Evoked (e) and spontaneous (s) AMPAR-mediated or NMDA-mediated EPSCs were recorded at a holding potential of $-60 \mathrm{mV}$ or $+40 \mathrm{mV}$, respectively. Synaptic responses were first identified around threshold stimulus. Then stimulus intensity was adjusted until failure responses of $50-60 \%$ at -60 $\mathrm{mV}$ to consecutive 60-200 trials were reached (Isaac et al., 1995; Liao et al., 1995). This final stimulus intensity was also used to evoke response at $+40 \mathrm{mV}$ for the same cells. All recordings, except where stated, were performed at room temperature $\left(22-24^{\circ} \mathrm{C}\right)$ for control and post-HS animals. All failure responses were visually checked and verified. Corresponding eEPSC failure rates at -60 and $+40 \mathrm{mV}$ and fraction of silent synapses $\left(1-\ln \left(F_{-60}\right) / \ln \left(F_{+40}\right)\right)$ were calculated using published methods (Liao et al., 1995).

For LTP studies, extracellular field potential recordings (fEPSPs) were used as previously described (Jensen et al., 1998) at $30-32^{\circ} \mathrm{C}$. The Schaffer collaterals from CA 3 to CA 1 were stimulated at 30 s intervals to obtain stimulus-response curves ( $0.3 \mathrm{~ms}$ stimulation duration; $0.1 \mathrm{~ms}$ duration was used for adult LTP recordings P60-P90). The control pathway was stimulated on the subiculum side of the recording pipettes. Intensity to evoke $50-60 \%$ maximal response was used as the test stimulus intensity for the fEPSP-LTP. Tetanus stimuli for fEPSP-LTP induction consisted of 200 shocks at $100 \mathrm{~Hz}$ in $2 \mathrm{~s}$ ( $20 \mathrm{~s}$ apart). Field EPSP slopes were used for fEPSP-LTP study (Jensen et al., 1998), unless otherwise specified. We also tested the eEPSC-LTP with pairing protocols at room temperature (to prevent washout at $30-32^{\circ} \mathrm{C}$ ) (Isaac et al., 1997). For eEPSC-LTP studies, cells were held at $10 \mathrm{mV}$ with 2 tetani $(0.3 \mathrm{~ms} 100 \mathrm{~Hz}$, separated 


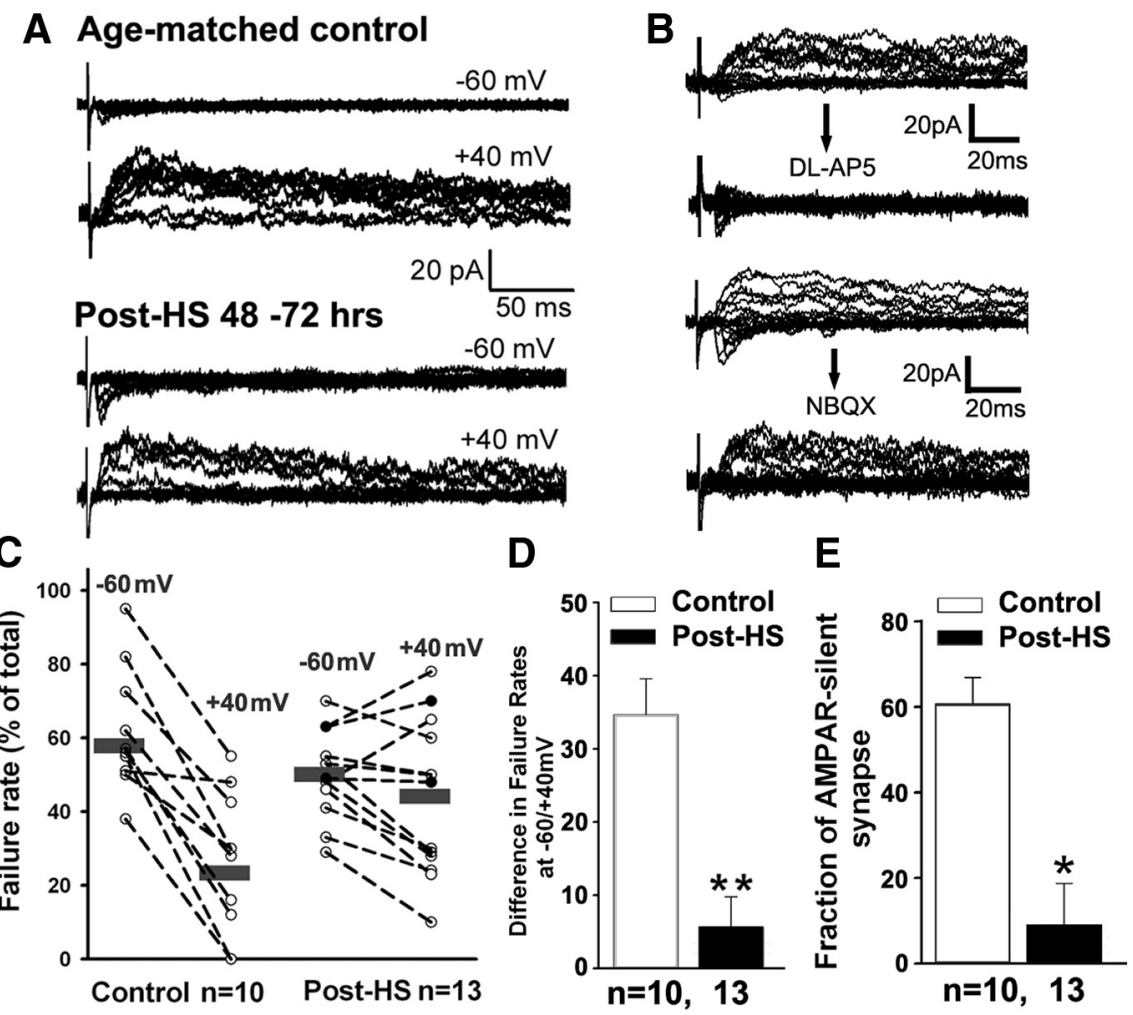

Figure 1. Attenuation in silent synapses following neonatal seizures. $\boldsymbol{A}$, Representative consecutive traces of evoked EPSC at $-60 \mathrm{mV}$ and $+40 \mathrm{mV}$ in CA1 pyramidal neurons from control (top two traces) and post-HS rats (in vivo seizures at P10) (bottom two traces). eEPS(s were recorded with whole-cell patch-clamp recordings and $60 \mu \mathrm{m}$ picrotoxin in ACSF at room temperature. $\boldsymbol{B}$, AMPARs mediate fast eEPSCs at $-60 \mathrm{mV}$ and NMDARs mediate slow decaying eEPSCs at $+40 \mathrm{mV}$. Top, Original consecutive traces show that $100 \mu \mathrm{m} \mathrm{DL}-A P 5$ in the ACSF completely blocked the slow-decaying eEPSCs at $+40 \mathrm{mV}$, leaving fast eEPSCs intact at -60 $\mathrm{mV}$ and $+40 \mathrm{mV}(n=5)$. In addition, failure rates for remaining eESPCs at -60 and $+40 \mathrm{mV}$ are very similar. Bottom, $20 \mu \mathrm{M}$ NBQX in the ACSF completely abolished the fast eESPCs at $-60 \mathrm{mV}$, leaving slow-decaying eEPSCs intact at $+40 \mathrm{mV}(n=5)$. Calibrations are labeled as indicated. $C$, Neonatal seizures decrease the failure rate difference between -60 and $+40 \mathrm{mV}$. $\boldsymbol{D}$, Failure rate difference between $-60 \mathrm{mV}$ and $+40 \mathrm{mV}$ is significantly lower in slices from rats $48-72 \mathrm{~h}$ after $\mathrm{HS}$ than age-matched controls. Data are expressed as mean \pm SEs. $n$ is cell number used for this analysis. ${ }^{* *} p<0.01 t$ test. $\boldsymbol{E}$, Fraction of silent synapses calculated with eEPSC failure rates is attenuated in post-HS group compared with controls. Silent synapse fraction was calculated with the equation $1-\ln \left(\right.$ FailureRate $\left._{-60}\right) / \ln \left(\right.$ FailureRate $\left._{+40}\right)$. Data are expressed as mean \pm SEs. $n$ is cell number used for this analysis. ${ }^{*} p<0.05$ by $t$ test.

at $20 \mathrm{~s}$ ) or $0 \mathrm{mV}$ with $2 \mathrm{~Hz}$ stimulus (200 shocks). The series resistance $(<15-20 \mathrm{M} \Omega)$ was monitored for stability during long recordings without significant changes $(<10-20 \%)$.

Data were collected using an Axopatch 200A amplifier (Molecular Devices) and Clampex 9.2 software (Molecular Devices) with compensation for series resistance (70\%) and cell capacitance, filtered at $2 \mathrm{kHz}$, and digitized at $20 \mathrm{kHz}$ using a Digidata $1320 \mathrm{~A}$ analog to digital converter (Molecular Devices). sEPSCs were detected automatically and frequency and amplitude histograms were constructed as described previously ( $\mathrm{Ra}-$ khade et al., 2008). Detected sEPSC events from each cell have similar rising (within $5 \mathrm{~ms}$ from $20 \%$ to $80 \%$ peak) and decay phases in the distribution plot, and these sEPSC events were aligned at their peak for peak-scaled nonstationary fluctuation analysis based on a previously described method (Benke et al., 1998). Baseline variance was obtained from episodes without synaptic events and was subtracted for final fitting analysis.

NBQX treatment. Rats were given an intraperitoneal injection of either saline vehicle or $20 \mathrm{mg} / \mathrm{kg}$ NBQX (Sigma) immediately and 12, 24, and $36 \mathrm{~h}$ after HS, then returned to their dam. This dosing was based on our previous study showing an antiepileptic effect of NBQX (Rakhade et al., 2008).

Statistics. Data were tested for normality using the Shapiro-Wilk normality test. Statistical significance was assessed using a Student's $t$ test, or a one-way ANOVA test when more than two groups were compared for data with normal distributions, and a MannWhitney or Wilcoxon matched-pairs signed rank test for comparisons of data that were not normally distributed. $p<0.05$ was considered statistically significant. All results were expressed as mean \pm SE unless specified.

\section{Results}

Decreases in silent synapses in hippocampal CA1 pyramidal neurons following early life HS

We have previously shown that seizures in the immature brain during the critical period of synaptogenesis induce rapid (within 1-3 h) posttranslational phosphorylation of AMPAR GluA1 Ser831 and Ser845 sites accompanied by increased AMPAR-mediated EPSC amplitude and frequency (Rakhade et al., 2008). Because increased phosphorylation of Ser831 and Ser845 enhances AMPAR conductance and membrane insertion, we hypothesized that this may subsequently change the fraction of silent synapses at later time points. Thus, we measured the ratio of silent NMDAR-only synapses to functional synapses containing AMPARs and NMDARs. Silent synapses are defined by a large failure rate of evoked EPSCs (eEPSCs) at negative membrane potentials ( -55 to $-65 \mathrm{mV}$, AMPAR-eEPSCs) concurrent with a small failure rate to the same stimuli at positive potentials $(+40$ mV, NMDAR-eEPSCs) (Liao et al., 1995; Marie et al., 2005). To measure the difference in failure rate between $-60 \mathrm{mV}$ and $+40 \mathrm{mV}$, we adjusted the stimulus intensity to set the failure rate at $-60 \mathrm{mV}$ to $50-60 \%$ $(99.87 \pm 13.91 \mu \mathrm{A}$ in post-HS, $n=13$ cells from 6 rats, $132.5 \pm 27.02 \mu \mathrm{A}$ in normoxia, $n=10$ cells from 5 rats, $t$ test, $p=0.286$ ), then examined the failure rates at $+40 \mathrm{mV}$ using the same stimulus intensity (Liao et al., 1995). Slices from age-matched P12-P13 controls exhibited the expected failure rate of $57.85 \pm 3.86 \%$ at $-60 \mathrm{mV}$ and a failure rate of $23.80 \pm 4.51 \%$ at $+40 \mathrm{mV}(n=10$ cells from 5 rats, Fig. $1 A$, top, $B, C$ ), yielding a failure rate difference of $34.05 \pm 4.72 \%$ (Fig. $1 D$ ), consistent with prior reports of a large population of silent synapses during this immature period (Isaac et al., 1995). In contrast, slices removed from rats $48-72 \mathrm{~h}$ after HS showed a failure rate of $50.08 \pm$ $3.22 \%$ at $-60 \mathrm{mV}$ and a failure rate of $44.12 \pm 5.72 \%$ at $+40 \mathrm{mV}$ ( $n=13$ cells from 6 rats, paired $t$ test, $p=0.12$; Fig. $1 A$, bottom, $C$ ), yielding a significantly decreased failure rate difference $(5.96 \pm$ $3.55 \%$ ) from controls ( $p=0.0001$, Fig. $1 C$ ). Accordingly, the calculated ratio of silent to functional synapses demonstrates that silent synapses in the post-HS group were significantly reduced $(9.08 \pm$ $11.60 \%, n=13$ cells from 6 rats) compared with controls $(60.90 \pm$ $6.75 \%, n=10$ cells from 5 rats, $t$ test, $p=0.006$; Fig. $1 E$ ). Finally, because NMDARs at this postnatal age exhibit some degree of $\mathrm{Mg}^{2+}$-insensitivity due to NR3A subunit expression (Zhou et al., 2009), we characterized these responses and found no effect of APV $(100 \mu \mathrm{M})$ on the failure rate, suggesting that $\mathrm{Mg}^{2+}$-insensitivity did not contribute to the failure rates at $-60 \mathrm{mV}$ at this age (data not shown). 


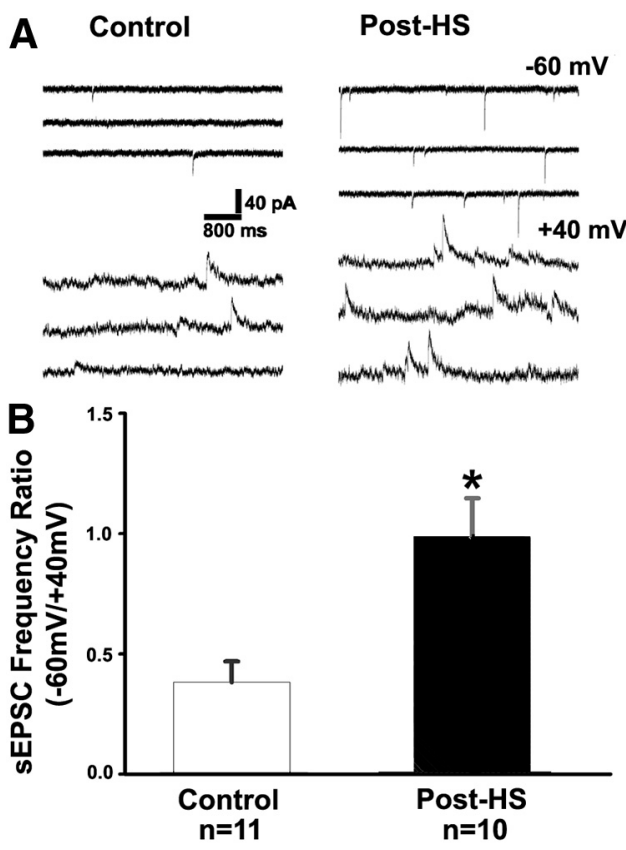

Figure 2. Decrease in silent synapses evidenced by increased AMPAR to NMDAR sEPSC frequency ratio following neonatal seizures $A$, Representative consecutive traces of spontaneous EPSCs at $-60 \mathrm{mV}$ (top) and $+40 \mathrm{mV}$ (bottom) in CA1 pyramidal neurons from control and post-HS groups. $B$, Summarized frequency ratio of sEPSCs at $-60 \mathrm{mV}$ to $+40 \mathrm{mV}$ for control and post-HS groups. Data are expressed as mean \pm SEs. $n$ is cell number used for this analysis. ${ }^{*} p<0.05$ by $t$ test.

An alternate measure of silent synapses is measurement of the glutamate-mediated spontaneous EPSC (sEPSC) frequency ratio between -60 and $+40 \mathrm{mV}$, with larger sEPSC frequency ratio indicating fewer silent synapses (Losi et al., 2002; Rumpel et al., 2004). This method is not sensitive to temperature (Losi et al., 2002) and therefore provides a good validation of failure rate measurements, which are more temperature sensitive. Consistent with our eEPSC failure rate analysis, glutamate receptormediated sEPSC frequency ratio at $-60 \mathrm{mV}$ to $+40 \mathrm{mV}$ was significantly larger in slices from rats $48-72 \mathrm{~h}$ after HS [0.95 \pm 0.17 (average $0.11 \pm 0.03 \mathrm{~Hz}$ at $-60 \mathrm{mV}$ and $0.13 \pm 0.03 \mathrm{~Hz}$ at $+40 \mathrm{mV}), n=10$ cells from 5 rats] compared with P12-P13 littermate controls $[0.41 \pm 0.07$ (average $0.05 \pm 0.01 \mathrm{~Hz}$ at $-60 \mathrm{mV}$ and $0.15 \pm 0.03 \mathrm{~Hz}$ at $+40 \mathrm{mV}), n=11$ cells from 6 rats, $t$ test $p=0.01$; Fig. 2]. These frequency data support the failure rate analysis indicating a significant decrease in NMDAR-only synapses.

In addition, to rule out the possible effects from hypoxia alone, we examined the silent synapse fraction in slices removed from rats that were exposed to hypoxia but that did not exhibit seizures ( $<5 \%$ of total of animals). First, slices removed at $48-72$ $\mathrm{h}$ after hypoxia in these rats did not show enhancement in amplitude or frequency of AMPA receptor-mediated sEPSCs compared with controls (Fig. $3 A$ ). In addition, differences in eEPSC failure rate $(55.23 \pm 1.68 \%$ at $-60 \mathrm{mV}$ and a failure rate of $19.25 \pm 4.42 \%$ at $+40 \mathrm{mV}$, yielding a failure rate difference of $35.98 \pm 3.84 \%, n=8$ cells from 3 rats, $t$ test, $p=0.76$; Fig. $3 C$ ) were similar to controls. Consistently, slices from hypoxic rats that did not exhibit seizures had no changes in sEPSC frequency ratio at $-60 \mathrm{mV}$ to $+40 \mathrm{mV}(0.47 \pm 0.04, n=6$ cells from 3 rats, $t$ test, $p=0.56$; Fig. $3 B$ ) compared with P12-P13 controls. These data suggest that seizure activity, not hypoxia alone, is required to exert these alterations in silent synapses.
Early life seizures increase colocalization of postsynaptic AMPARs and NMDARs in CA1 s. radiatum

Given the electrophysiological evidence for a decrease in NMDAR-only containing synapses after in vivo $\mathrm{HS}$ at $\mathrm{P} 10$, we next examined the synaptic colocalization of NMDAR and AMPAR subunits at $48-72 \mathrm{~h}$ after HS. These measurements were made in CA1 s. radiatum, as this was the area containing the synapses from which the electrophysiological data were gathered (Fig. 4A,B). Because the difference between active and silent synapses is the presence of AMPARs, we triple-immunolabeled for GluA1 (the most common AMPAR subunit at this developmental stage in the hippocampus), NR1 (the obligate subunit for NMDARs), and the presynaptic marker synaptophysin (Fig. $4 C, D)$. We then assessed how many of the NR1 puncta were in contact with synaptophysin puncta, and of those how many were also in contact with GluA1 puncta. In slices removed from rats post-HS, the percentage of puncta containing only NR1/synaptophysin [without GluA1 colocalization, in contact with synaptophysin puncta, $6.4 \pm 1.3 \%$ (median $=3.3)$ ] in s. radiatum was decreased by $\sim 43 \%$ compared with age-matched controls $[11.2 \pm 1.3 \%$ (median $=9.8), n=18$ fields in 6 rats/group; Mann-Whitney $p=0.002$; Fig. $4 E]$. We verified the relative decrease in colocalization of NR1 with GluA1 by examining multiple thresholds of the GluA1 image in a small, randomly chosen subset of images, and determined that the increased colocalization was not an artifact of threshold level $(n=3$ fields per group per threshold level, $p=0.016$ by Wilcoxon matched-pairs signed rank test; Fig. $4 F$ ) (Nie et al., 2010). In addition, we did not detect a change in the total number of synaptophysin/NR1 puncta [Fig. $4 G ; 2.1 \pm 0.17($ median $=2.6)$ puncta $/ \mu \mathrm{m}^{2}$ in $n=19$ fields from 6 rats with seizures; $2.4 \pm 0.20($ median $=2.4)$ puncta $/ \mu \mathrm{m}^{2}$ in $n=18$ fields from 6 control rats, Mann-Whitney test, $p=0.33$ ]. Consistent with the electrophysiological data, these results suggest HS decrease silent synapses. The increased colocalization of GluA1 and NR1 subunits is occurring at a time point immediately following a rapid, seizure-induced increase in phosphorylation of GluA1 Ser831 and 845, which is known to increase trafficking of AMPAR GluA1 subunits to the synaptic membrane and increase synaptic efficacy (Esteban et al., 2003).

\section{Increase in synaptic GluA1 following HS}

Given the increased colocalization of GluA1 and NR1 subunits, we next wanted to determine whether there was a net increase in GluA1 in addition to the aforementioned decrease in the fraction of silent synapses. Using blinded quantitative immunocytochemistry in a subset of fields used for the above NR1/GluA1/synaptophysin colocalization analysis, we observed a 53\% increase post-HS compared with controls in the total number of synaptophysin/GluA1 puncta $\left[1.5 \pm 0.15(\right.$ median $=1.4)$ puncta $/ \mu \mathrm{m}^{2}$ in controls and $2.3 \pm 0.13($ median $=2.5)$ puncta $/ \mu \mathrm{m}^{2}$ post-HS in 9 fields from 5 rats/group, Mann-Whitney $p=0.001$ ] in a random subset of the same fields in which we analyzed NR1/GluA1/ synaptophysin overlap (Fig. $4 H$ ). These data support the idea that HS increases the incorporation of AMPAR subunits at glutamatergic synapses that contain NMDARs and are consistent with the increase in AMPAR-mediated sEPSCs.

To confirm these structural data, we conducted a peak-scaled nonstationary fluctuation analysis (Benke et al., 1998) to examine changes in AMPAR number at single synapses in CA1 pyramidal neurons at $48-72 \mathrm{~h}$ after HS. In this analysis, all sEPSC events selected from each neuron showed similar rising and decay phases, which indicates that there was no significant space-clamp filter effect arising from differences in spatial distribution of 


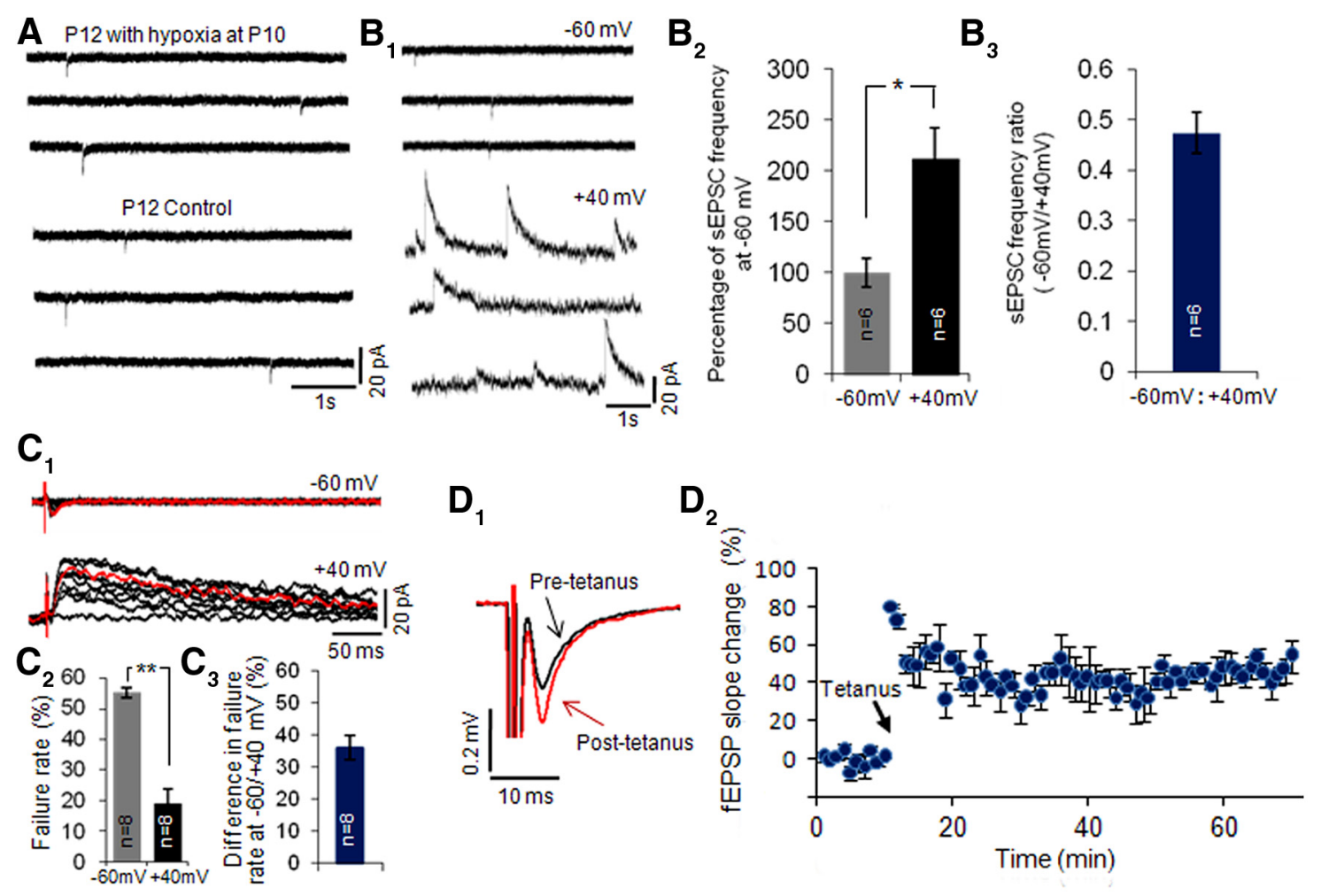

Figure 3. Hypoxia alone did not affect AMPAR function, silent synapses and LTP. A, Representative consecutive traces of spontaneous EPSCs at $-60 \mathrm{mV}$ in CA1 pyramidal neurons from control and posthypoxia with no seizures groups. $\boldsymbol{B}$, Hypoxia alone did not decrease silent synapses evidenced by similar AMPAR to NMDAR sEPSC frequency ratio compared with controls. $\boldsymbol{B}_{1}$, Representative consecutive traces of spontaneous EPSCs at $-60 \mathrm{mV}$ (top) and $+40 \mathrm{mV}$ (bottom) in CA1 pyramidal neurons from the posthypoxia with no seizures group. $\boldsymbol{B}_{2}$, Summarized frequency of sEPSCs at $-60 \mathrm{mV}$ and $+40 \mathrm{mV}$. $\boldsymbol{B}_{3}$, Summarized frequency ratio of sEPSCs at $-60 \mathrm{mV}$ to $+40 \mathrm{mV}$. C, No detectable change in silent synapse following hypoxia alone. $\boldsymbol{C}_{1}$, Typical consecutive traces of evoked EPSC at $-60 \mathrm{mV}$ (top) and $+40 \mathrm{mV}$ (bottom) in CA1 pyramidal neurons from posthypoxia with no seizures rats. $C_{2}$, Summarized failure rate of evoked EPSCs at $-60 \mathrm{mV}$ and $+40 \mathrm{mV}$. $\boldsymbol{C}_{3}$, Summary of the failure rate difference of evoked EPSCS at $-60 \mathrm{mV}$ and $+40 \mathrm{mV}$. D, LTP is not altered by hypoxia alone. $\boldsymbol{D}_{1}$, Representative averaged fEPSP traces recorded in stratum radiatum of CA1 in response to $S$ chaffer collateral stimulation at $30 \mathrm{~s}$ interval. Black traces show averaged recordings before tetanus stimulation. Red traces show averaged recordings at 40 min after tetanus. $\boldsymbol{D}_{2}$, Time course of fEPSP change following tetanus stimulation ( $2 \times$ tetanus at $100 \mathrm{~Hz}$ for $1 \mathrm{~s}$, with $20 \mathrm{~s}$ interval) shows significant potentiation in hypoxia with no seizures group ( $n=3$ slices from 3 rats). Arrows mark the starting point of tetanus stimulation. Data are expressed as mean \pm SEs. $n$ is cell number used for this analysis. ${ }^{*} p<0.05$ and ${ }^{* *} p<0.01$ by paired $t$ test.

sEPSC events (Fig. 5A, B). The estimated number of AMPARs per synapse post-HS ( $19.64 \pm 1.56, n=15$ cells from 8 rats) was significantly increased above P12-P13 controls (12.03 \pm 1.17 , $n=14$ cells from 7 rats, $t$ test, $p=0.01$; Fig. $5 C$ ). This increase in estimated number of AMPARS was accompanied by a change in single-channel conductance of synaptic AMPARs $(24.45 \pm 2.41$ $\mathrm{pS}, n=15$ cells from 8 rats for post-HS vs $17.48 \pm 1.67 \mathrm{pS}, n=14$ cells from 7 rats for control, $t$ test $p=0.04$; Fig. 5D). The increase of AMPARs at synapses is consistent with their role in the decrease in silent NMDAR-only synapses at $48-72 \mathrm{~h}$ following hypoxia-induced seizures.

\section{Early life seizures result in acute and long-term impairment in hippocampal LTP}

The conversion of silent to unsilenced synapses by incorporation of AMPARs has been suggested to be involved in certain forms of synaptic plasticity expression associated with learning and memory, such as LTP, and with the genesis of functional synapses during early development (Petralia et al., 1999; Busetto et al., 2008; Kerchner and Nicoll, 2008). Therefore, we hypothesized that LTP would become attenuated $48-72 \mathrm{~h}$ after HS, as the pool of silent synapses becomes depleted. We thus assessed LTP in hippocampal slices removed from rats at $48-72 \mathrm{~h}$ after in vivo $\mathrm{HS}$ by extracellular recordings of fEPSPs in area CA1. Schaffer collateral tetanic stimulation was 50\% maximal stimulus amplitude based on input/output curves. Initial baseline fEPSP amplitudes in CA1 from post-HS rats $(0.62 \pm 0.08 \mathrm{mV}, n=6$ slices in 6 rats $)$ were significantly larger than those of controls $(0.40 \pm 0.07 \mathrm{mV}$, $n=6$ slices in 6 rats, $t$ test, $p=0.031$; Fig. $6 A$ ) without significant differences in fiber volley amplitude between groups (post-HS = $0.12 \pm 0.02 \mathrm{mV}, n=6$ slices in 6 rats, control $=0.11 \pm 0.01 \mathrm{mV}$, $n=6$ slices in 6 rats, $t$ test $p=0.95)$. Following tetanic stimulation, the potentiation of fEPSP slopes was significantly attenuated in amplitude and duration in post-HS slices (potentiated by $6.18 \pm 3.12 \%, n=5$ slices from 5 rats, at $40 \mathrm{~min}$ ) compared with those from P12-P13 controls (potentiated by $60.69 \pm 23.06 \%$, $n=6$ slices from 6 rats, at $40 \mathrm{~min}, t$ test $p=0.03$; Fig. $6 \mathrm{B1}$ ). This stimulation did not result in potentiation in control pathways in either group (Fig. 6B2). In addition, the slices from HS animals did not show any changes compared with control slices in CA1 pyramidal neuron resting membrane potential, input resistance, and action potential amplitude and threshold (Table 1).

Next, we confirmed our fEPSP LTP findings by examining potentiation of evoked EPSCs (eEPSCs) using whole-cell recordings and a pairing protocol to synchronize presynaptic stimulation and postsynaptic neuronal depolarization $(+10 \mathrm{mV})$ (Liao et al., 1995). With a stimulus calibrated to a $50 \%$ success rate of eEPSCs, eEPSC amplitudes were larger in slices removed at $48-72 \mathrm{~h}$ after HS compared with P12-P13 controls. The pairing protocol resulted in significantly less potentiation of eEPSCs with a smaller degree in post-HS groups $(97.62 \pm 4.52, n=6$ slices in 6 rats) compared with those in slices from control rats (190.99 \pm 
11.17, $n=6$ slices in 6 rats; Fig. $6 C, D)$. Thus, at $48-72 \mathrm{~h}$ after HS, LTP was attenuated as determined by both fEPSP and eEPSC measurements from tetanic and paired-protocol stimulation paradigms (Liao et al., 1995), respectively. In contrast, no deficits in fEPSP-LTP were observed in hippocampal slices from animals exposed to hypoxia that did not exhibit seizures compared with normoxic controls (potentiated by $50.43 \pm 6.69 \%, n=3$ slices from 3 rats, at 40 min after tetanus, $t$ test, $p=0.77$; Fig. $3 D$ ), suggesting that hypoxia alone did not influence the LTP expression in our model.

To confirm that these changes in LTP were not due to a presynaptic change, we examined paired-pulse responses in all slices. In slices removed at $48-72 \mathrm{~h}$ after seizures, paired-pulse facilitation (pairedpulse intervals of $30,50,70,90,200$, and $400 \mathrm{~ms}$ ) did not show any significant changes from P12-P13 controls $(n=7$ slices from 7 rats per group, $t$ test, all $p>$ 0.05 ; Table 2 ), supporting a postsynaptic mechanism of AMPAR alteration and impairment and occlusion of LTP following HS.

As an additional analysis, we examined the coefficients of AMPAR- and NMDAReEPSC amplitude variance, as LTP has been shown to result in a decrease in the coefficient of AMPAR variance without a change in the coefficient of NMDAR variance (Kullmann, 1994; Marie et al., 2005). We hypothesized that HS would result in a similar change as that reported for LTP. In control P12-P13 rats under baseline conditions, the coefficient of AMPAR-eEPSC variance $(0.29 \pm 0.02, n=7$ cells from 3 rats, paired $t$ test, $p=0.01$ ) in CA1 pyramidal neurons was always larger than that of NMDAR-eEPSC variance $(0.15 \pm 0.04$, $n=7$ cells from 3 rats, isolated with $20 \mu \mathrm{M}$ $\mathrm{NBQX}$, at $+40 \mathrm{mV})$. However, in slices removed at $48-72 \mathrm{~h}$ after HS, the coefficient of AMPAR-eEPSC variance $(0.25 \pm$ $0.02, n=8$ cells from 4 rats, paired $t$ test, $p=0.37)$ was very similar to NMDAReEPSC variance $(0.22 \pm 0.02, n=8$ cells from 4 rats). These data indicate a decrease in the AMPAR coefficient of variance following $\mathrm{HS}$, supporting the hypothesis that HS shares mechanisms with and can at least in part occlude LTP.

While we show here that at least one consequence of HS is a subacute attenuation in LTP $48-72 \mathrm{~h}$ after HS, we have previously reported an enhancement of LTP immediately (within $10 \mathrm{~min}$ ) after seizures (Jensen et al., 1998). We hypothesized that this time-dependent difference in effects on LTP was due to changes in both phosphorylation state and silent syn-
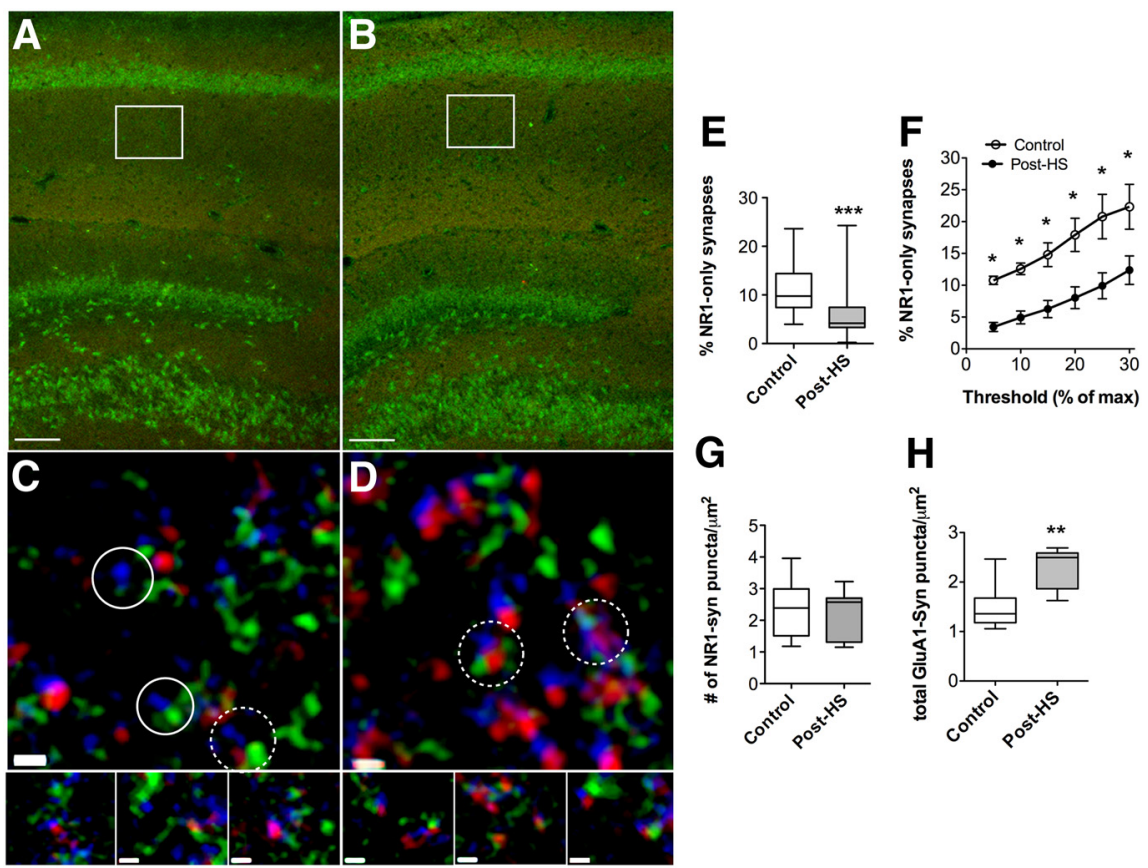

Figure 4. NR1-only synapses decrease $48 \mathrm{~h}$ after seizure. $A-D$, Examples of sections from $\mathrm{P} 12$ control $(A, C)$ and $48 \mathrm{~h}$ post-HS rats $(\boldsymbol{B}, \boldsymbol{D})$, immunolabeled for NR1 (green), GluA1 (red), and synaptophysin (blue, $\boldsymbol{C}$ and $\boldsymbol{D}$ only), from CA1 s. radiatum showing similar tissue quality for control and post-HS. Boxes in $\boldsymbol{A}$ and $\boldsymbol{B}$ represent areas imaged. In $\boldsymbol{C}$ and $\boldsymbol{D}$, solid circles indicate NR1/Synonly labeling and dotted circles indicate NR1/GluA1/Syn labeling (with digitally zoomed-in areas below to better show individual puncta). Scale bars: $\boldsymbol{A}, \boldsymbol{B}, 100 \mu \mathrm{m} ; \boldsymbol{C}, \boldsymbol{D}, 0.5 \mu \mathrm{m}$. $\boldsymbol{E}$, The percentage of NR1-only synapses was lower $48 \mathrm{~h}$ after HS than in age-matched controls, indicating a decrease in silent synapses after seizures; $p=0.002$ by Mann-Whitney test. $F$, In a subset of fields, the percentage of NR1-only synapses was calculated at increasing thresholds to rule out threshold artifact. $p=0.016$ by Wilcoxon matched-pairs signed rank test. $G$, Quantification of the total number of NR1/synaptophysin-labeled puncta/ $\mu \mathrm{m}^{2}$ shows no change from controls in rats $48 \mathrm{~h}$ after HS. $p=0.33$ by Mann-Whitney test. $\boldsymbol{H}$, In a subset of fields, we measured total GluA1/synaptophysin-labeled puncta/ $\mu \mathrm{m}^{2}$ and found they were significantly increased at $48 \mathrm{~h}$ after HS over that of controls. ${ }^{*} p<$ $0.05,{ }^{* *} p<0.01,{ }^{* * *} p<0.001$ by Mann-Whitney test.
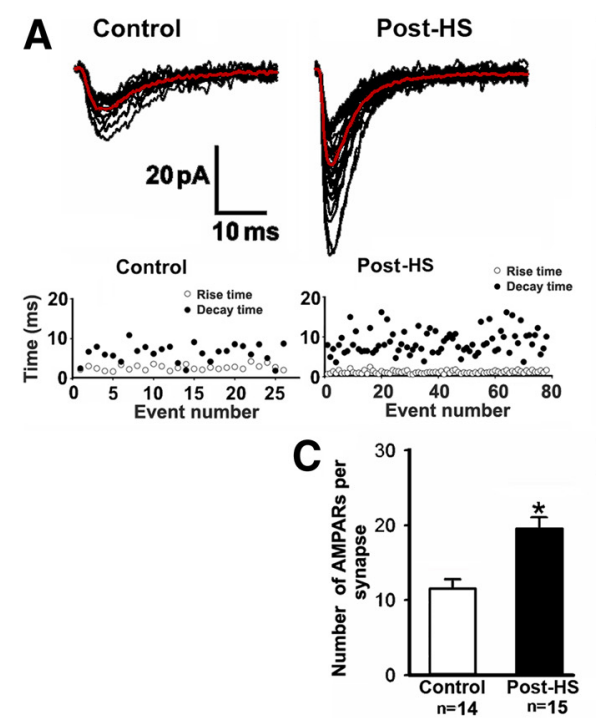
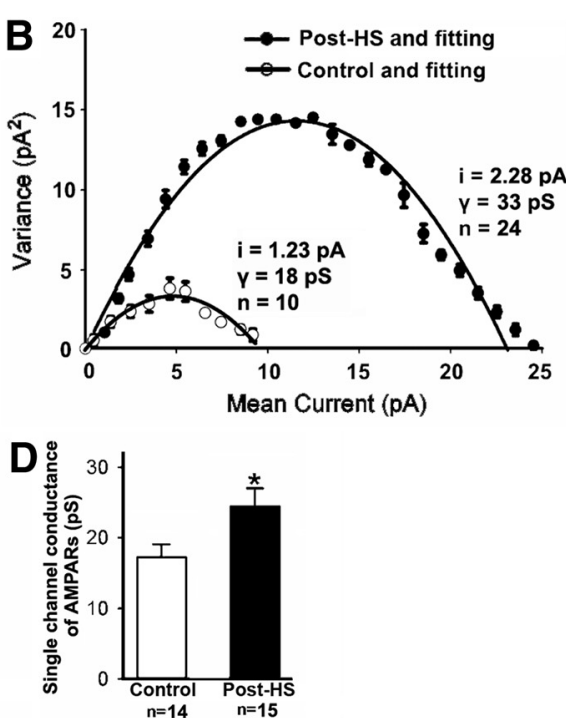

Figure 5. Increase in synaptic AMPAR number following neonatal seizures. $\boldsymbol{A}$, Representative spontaneous EPSC events recorded at $-60 \mathrm{mV}$ in CA1 pyramidal neurons from control (left) and post-HS groups (right). The events used in this analysis for each neuron were selected by their similar rising (within 5 ms rise time $20 \%$ to $80 \%$ peak) and decay phases (bottom). Red traces represent the corresponding average of these events. $\boldsymbol{B}$, Representative fitted curves for one control neuron and one neuron from a post-HS rat with peaked-scaled nonstationary fluctuation analysis. Summarized fitted individual values are shown on the side of corresponding curves. Data are expressed as mean \pm SD. $i$ indicates single-channel current, $\gamma$ indicates single-channel conductance, and $n$ indicates AMPAR number per synapse. C, Summarized AMPAR number per synapse for control and post-HS. Data are expressed as mean \pm SEs. $n$ is cell number used for this analysis. ${ }^{*} p<0.05$ by $t$ test. $\boldsymbol{D}$, Summarized AMPAR single-channel conductance for control and post-HS. Data are expressed as mean \pm SEs. $n$ is cell number. ${ }^{*} p<0.05$ by $t$ test. 
A

Control

Post-HS
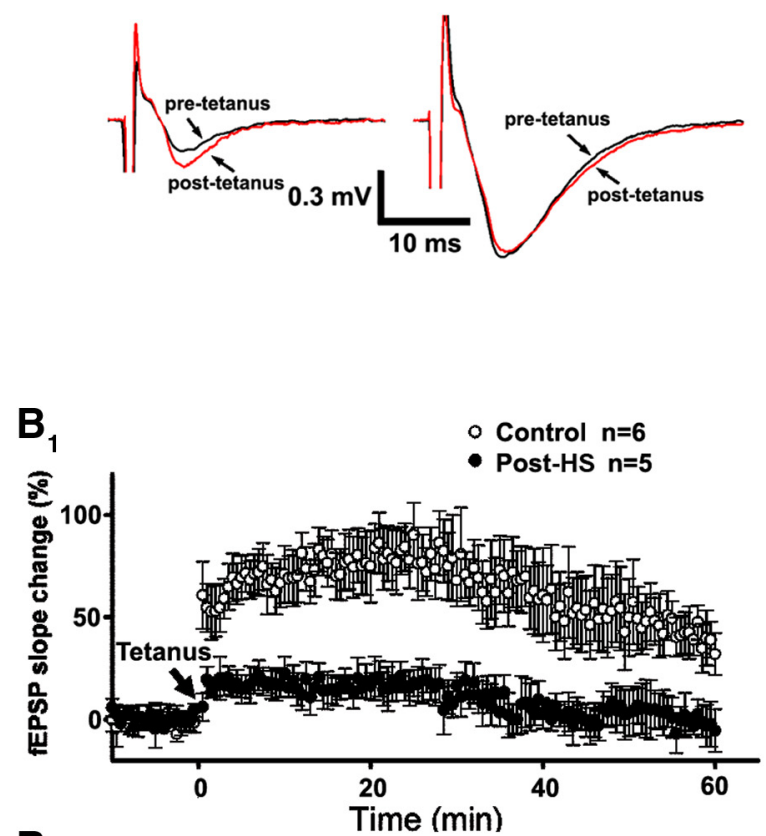

$B_{2}$

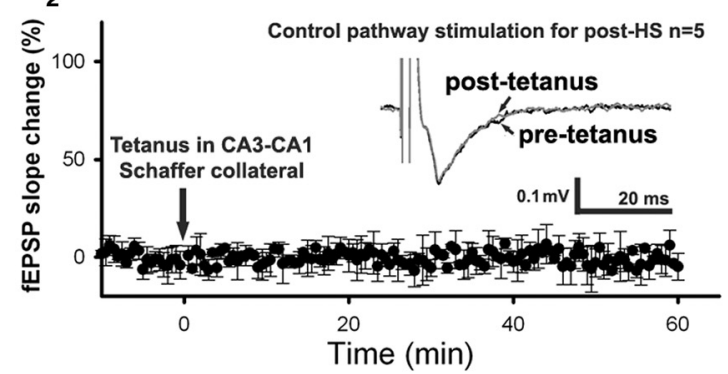

$C_{1} \quad$ Control

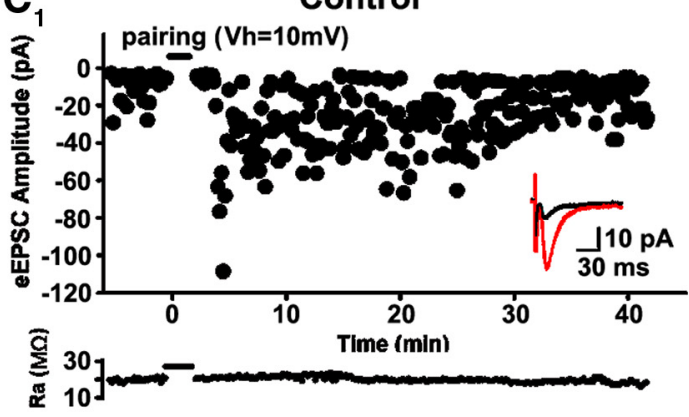

$\mathrm{C}_{2} \quad$ Post-HS

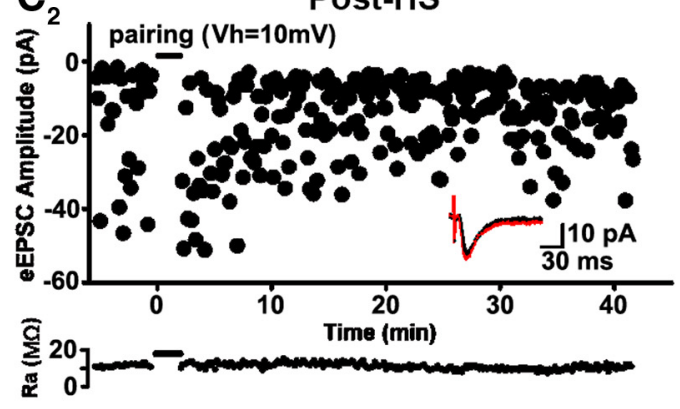

D

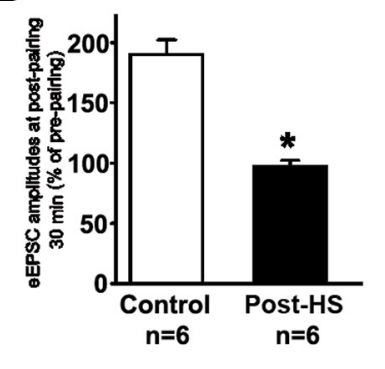

Figure 6. Decrease in LTP following neonatal seizures. A, Representative averaged fEPSP traces recorded in CA1 pyramidal neurons from control (left) and post-HS groups (right) with Schaffer collateral from CA3 to CA1 stimulated at 30 s interval. Black traces show averaged recordings before tetanus stimulation. Red traces show averaged recordings at 40 min after tetanus. $\boldsymbol{B}_{1}$, Time course of tetanus stimulus-induced fEPSP change shows attenuated LTP in post-HS compared with control LTP. Data are expressed as mean \pm SEs. $n$ is slices used for analysis. Arrow marks the starting point of tetanus stimulation ( $2 \times$ tetanus at $100 \mathrm{~Hz}$ for $1 \mathrm{~s}$, with $20 \mathrm{~s}$ interval). $\boldsymbol{B}_{2}$, The same stimulation of the control pathway does not induce LTP. $C$, Time course of eEPSC amplitude change in CA1 pyramidal neurons with pairing protocol shows attenuation in LTP enhancement for post-HS $\left(\boldsymbol{C}_{2}\right)$ compared with control $\left(\boldsymbol{C}_{\mathbf{7}}\right)$. Whole-cell patch-clamp recordings were used with the pairing protocol. Insets are representative averaged eEPSC traces and calibrations are indicated as labeled. The pairing was applied by synchronizing presynaptic stimulation (200 shocks in $2 \mathrm{~Hz}$ or $100 \mathrm{~Hz}$ tetanus) and postsynaptic depolarization $(+10 \mathrm{mV})$. On bottom is the corresponding time course of access resistance along whole experiments. D, Summarized eEPSC amplitude changes before pairing and 30 min after pairing for control and postseizure groups. Data are expressed as mean \pm SEs. $n$ is slices used for this analysis. ${ }^{*} p<0.05$ by $t$ test.

Table 1. Quantitative comparison of CA1 pyramidal neuronal properties

\begin{tabular}{lrr}
\hline & \multicolumn{1}{c}{ Age-matched control } & \multicolumn{1}{c}{ Post-HS at 48-72 h } \\
\hline Resting membrane potential $(\mathrm{mV})$ & $-61.38 \pm 0.68(n=8)$ & $-60.3 \pm 1.75(n=8)$ \\
Input resistance $(\mathrm{M} \Omega)$ & $326.72 \pm 45.08(n=7)$ & $285.64 \pm 25.14(n=8)$ \\
AP amplitude $(\mathrm{mV})$ & $105.69 \pm 5.75(n=6)$ & $104.37 \pm 7.91(n=6)$ \\
AP tThreshold $(\mathrm{mV})$ & $48.78 \pm 1.16(n=6)$ & $47.77 \pm 0.93(n=6)$ \\
\hline
\end{tabular}

Comparison between neurons from slices made $48-72 \mathrm{~h}$ post-HS and from age-matched controls (see Results). Input resistance is measured with potassium-based intracellular solution; action potential (AP) amplitude is measured from 10 to $90 \%$ of APs. AP threshold is the membrane potential at which $d V / d t$ is larger than $0.3 \mathrm{mV} / \mathrm{ms}$. Values are mean $\pm \mathrm{SEM} ; n$ is the cell number used.

apse fraction between the acute ( $1-3 \mathrm{~h})$ and subacute $(48-72 \mathrm{~h})$ periods after seizure. Immediately after seizures, synaptic efficacy is transiently heightened due to GluA1 Ser831 and Ser845 phosphorylation, but returns to control levels by $48 \mathrm{~h}$ (Rakhade et al., 2008). Indeed, we conducted an identical failure rate analysis to that reported above (Fig. 1D) for this early time point of 1-3 h. The calculated ratio of silent to functional synapses in the post-HS group was only moderately reduced $(35.9 \pm 13.26 \%$, $n=6$ from 3 rats) compared with normoxic controls $(73.8 \pm$
Table 2. Comparison of paired-pulse facilitation

\begin{tabular}{llll}
\hline Interval (ms) & Age-matched control $(n=7)$ & $48-72 \mathrm{~h}$ postseizure $(n=7)$ & $t$ test value \\
\hline 30 & $2.03 \pm 0.09$ & $2.48 \pm 0.19$ & $p=0.067$ \\
50 & $2.04 \pm 0.14$ & $2.09 \pm 0.16$ & $p=0.823$ \\
70 & $1.99 \pm 0.16$ & $2.56 \pm 0.27$ & $p=0.110$ \\
90 & $2.07 \pm 0.14$ & $2.25 \pm 0.19$ & $p=0.562$ \\
200 & $1.77 \pm 0.16$ & $1.71 \pm 0.19$ & $p=0.830$ \\
400 & $1.34 \pm 0.07$ & $1.36 \pm 0.09$ & $p=0.863$ \\
\hline
\end{tabular}

There is no significant change in paired-pulse facilitation at any interval between slices from rats $48-72 \mathrm{~h}$ post-HS and age-matched controls ( $t$ test, $p>0.05$, see Results). Values are means \pm SEM; $n$ is the cell number used for this analysis.

$7.25 \%, n=7$ from 3 rats), unlike the almost complete depletion $(9 \%$, Fig. $1 D)$ seen at $48-72 \mathrm{~h}$ when LTP is highly attenuated. Thus, the potential for synaptic plasticity is markedly different at the immediate time point versus the subacute $48-72 \mathrm{~h}$ time point we report above.

As these results suggest that synaptic plasticity is dysregulated early following neonatal HS, we next assessed whether there also 
were long-term changes in the capacity of hippocampal neurons for LTP in adult rats after HS. In slices removed from P60 animals after HS at P10, fEPSP-LTP was markedly decreased following Schaffer collateral stimulation $(10.14 \pm 18.34 \%$, $n=6)$ compared with naive adult controls $(73.80 \pm 14.46 \%, n=6, t$ test, $p=$ 0.021 at 40 min after tetanus; Fig. $7 B$ ). The long-term changes in LTP are consistent with neurobehavioral data showing impaired learning and water maze performance in adulthood following P10 HS (Mikati et al., 2005).

Postseizure NBQX treatment in vivo attenuates subsequent seizure-induced alterations in LTP

Given that we have previously shown that post-HS treatment with AMPAR antagonists reverses the HS-induced enhancement of AMPAR and the long-term increases in seizure susceptibility (Jensen et al., 1995; Koh et al., 2004; Rakhade et al., 2008), we investigated whether this treatment would also prevent HS-induced loss of silent synapses and impaired LTP. Whole-cell recordings were performed in hippocampal CA1 pyramidal neurons in slices removed (at $48-72 \mathrm{~h}$ ) from rats with $\mathrm{P} 10 \mathrm{HS}$ treated immediately and 12,24 , and $36 \mathrm{~h}$ after seizures with either saline vehicle or $20 \mathrm{mg} / \mathrm{kg}$ NBQX, as per our previously described protocols (Rakhade et al., 2008). In slices removed at $48-72 \mathrm{~h}$ after HS from rats with NBQX after treatment, eEPSCs in CA1 pyramidal neurons showed a larger failure rate at $-60(73.86 \pm 4.78)$ than at $+40 \mathrm{mV}(37.38 \pm 5.28)$ at $48-72 \mathrm{~h}$ after HS (paired $t$ test, $p=0.0002, n=7$ cells from 4 rats; Fig. $8 \mathrm{~A}$ ), similar to those of naive controls (Fig. $8 \mathrm{C}$ ), but significantly greater than untreated rats following $\mathrm{HS}$ ( $t$ test, $p=0.004$; Fig. $8 C$ ). As this was a postseizure treatment, seizure counts did not differ during hypoxia between the groups $(7.62 \pm 0.42, n=13$ rats for NBQX treatment group vs $8.22 \pm 0.63, n=8$ rats for vehicle group, $t$ test, $p=0.417$ ). Importantly, the brief dosing regimen of NBQX itself did not significantly influence the silent synapse number or development in controls, suggesting safety (Fig. $8 \mathrm{C}$ ). These data indicate that NBQX treatment could reverse HS-induced loss of silent synapses.

Finally, we examined whether NBQX treatment following P10 HS also protected against changes in LTP. Induction of LTP by tetanic stimulation of Schaffer collaterals from CA3 to CA1 in slices removed at $48-72 \mathrm{~h}$ after HS with NBQX treatment (fEPSP-LTP) showed similar potentiation $(57.75 \pm 11.97 \%, n=$ 6 slices from 6 rats) to P12-P13 controls ( $t$ test, $p=0.758$ ) (Fig. $8 B$ ), and was significantly greater than the impaired LTP seen in untreated rats post-HS ( $t$ test, $p=0.0064$ at 40 min after tetanus; Fig. 6B). In addition, in slices removed from P60 animals post-HS with NBQX treatment, fEPSP was markedly potentiated following Schaffer collateral stimulation (83.96 $\pm 13.86 \%$ of slope change at 40 min after tetanus, $n=5$ slices; Fig. $8 D$ ), which was similar to P60 controls ( $t$ test, $p=0.629$ ) and significantly larger than that seen in P60 untreated rats post-HS ( $t$ test, $p=$ 0.0128). Notably, NBQX treatment alone (no HS) in control rats did not alter LTP at both P12-P13 and P60 (data not shown).
Adult post seizures

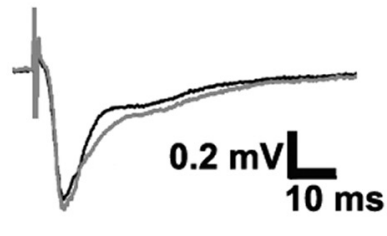

- Control $n=6$

- Post-seizures $n=6$

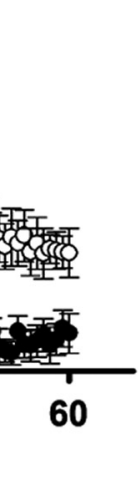

20

60

Figure 7. Adult LTP was also decreased following neonatal seizures. $\boldsymbol{A}$, Representative averaged fEPSP traces recorded at $32^{\circ} \mathrm{C}$ CA1 pyramidal neurons from adult control (left, top) and adult post-HS (in vivo seizures at P10) (right, top) with Schaffer number used for this analysis. Arrow marks the starting point of tetanus stimulation.

Together, these results suggest that postseizure treatment with AMPAR antagonists may prevent the HS-induced attenuation of silent synapses and subsequent alteration of LTP.

\section{Discussion}

Cognitive impairment following early life seizures is an increasingly appreciated problem in patients with epilepsy, yet the mechanisms whereby these seizures can cause intellectual and memory impairment are unknown. Here, we explored the hypothesis that early life seizures disrupt early patterns of synaptic development and impair network plasticity. We used a model of HS, the most clinically common cause of neonatal seizures. We demonstrated a decrease in the proportion of silent to active synapses by $48-72 \mathrm{~h}$ post-HS in CA1, as evidenced by decreases in eEPSC failure rate difference and increases in AMPAR to NMDAR-sEPSC frequency ratio. Consistently, we found decreased NMDAR-only synapses and increased colabeling of NMDAR and AMPAR markers in CA1 s. radiatum. The decrease in silent synapses was associated with a concurrent attenuation of LTP. Treatment with AMPAR antagonist NBQX has previously proven effective against HS-induced changes in acute and chronic neuronal and network excitability (Jensen et al., 1995) and increased seizure susceptibility (Koh et al., 2004). Here, we show the same postseizure NBQX treatment protocol can reverse alterations in silent synapses and LTP when administered following the initial seizures. These data suggest a possible mechanism of HS-induced synaptic dysplasticity that may contribute to cognitive consequences of epilepsy during the early developmental critical period of synaptogenesis, and that this may be a reversible process. 
A

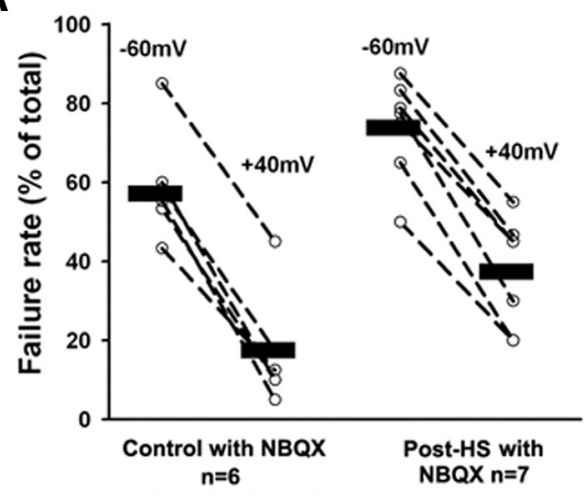

B

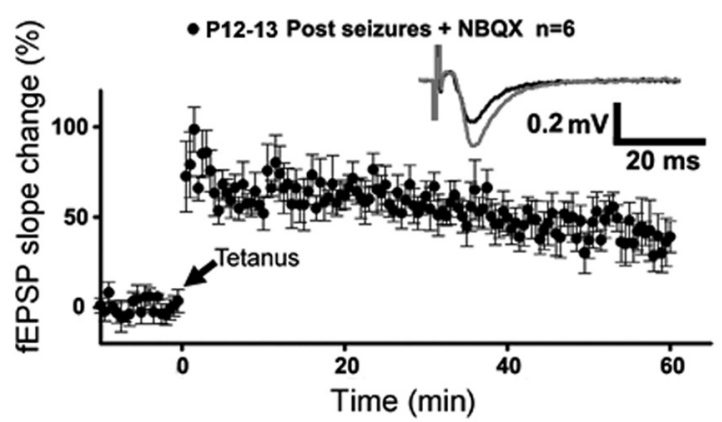

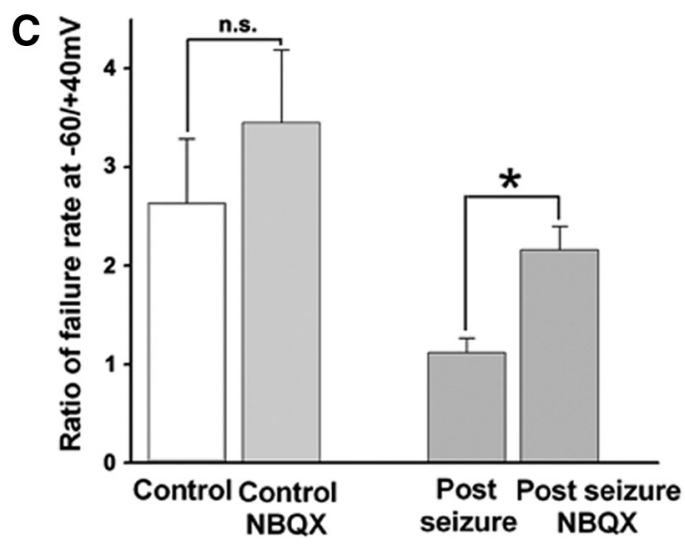

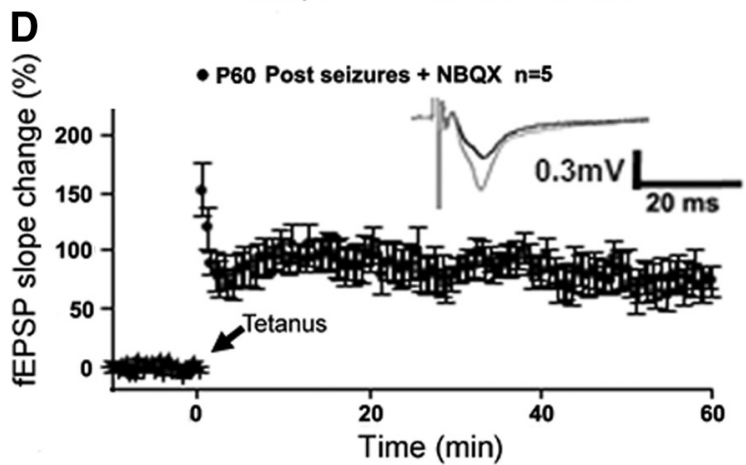

Figure 8. Postseizure treatment with NBQX reversed the decrease in silent synapse and subacute attenuation of $L T P$. $A$, Failure rates of individual neurons and summarized eEPSC failure rate ratio at $-60 \mathrm{mV}$ to $+40 \mathrm{mV}$ (linked by a dashed line) for control + NBQX and post-HS +NBQX ( $p=0.0002$ by paired $t$ test in post-HS cells). Data are expressed as mean \pm SEs. $n$ is cell number used for analysis. $\boldsymbol{B}$, Time course of tetanus stimulus-induced fEPSP enhancement in slices from P12-P13 post-HS NBQX rats shows very similar LTP as slices from controls ( $p=0.758$ by $t$ test). Data are expressed as mean \pm SEs; $n$ is slice number used for this analysis. Arrow marks the starting point of tetanus stimulation. Inset, Representative averaged fEPSP traces with a black trace as the averaged baseline recordings before tetanus stimulation, and a gray trace as the averaged recordings at 40 min posttetanus stimulation. $C$, Averaged failure rate ratios for control + NBQX are very similar to that of controls. However, eEPSC failure rate ratios in slices from post-HS + NBQX rats are increased compared with the post-HS + vehicle group $\left({ }^{*} p<0.05\right.$ by $t$ test). Data are expressed as mean \pm SEs. $n$ is cell number used for this analysis. $D$, Time course of tetanus stimulus-induced fEPSP enhancement in slices from P60 post-HS + NBQX rats shows very similar LTP as slices from controls ( $p=$ 0.629 by $t$ test). Data are expressed as mean \pm SEs. $n$ is slice number used for this analysis. Arrow marks the starting point of tetanus stimulation. Inset, Representative averaged fEPSP traces with a black trace as the averaged baseline recordings before tetanus stimulation and a gray trace as the averaged recordings at 40 min posttetanus stimulation.

\section{Neonatal seizures can decrease silent synapses}

During the early neonatal period, NMDAR-only, so-called silent, synapses are prevalent throughout the CNS, including the hippocampus, but decrease by the third postnatal week in most brain areas (Isaac et al., 1995; Liao et al., 1995; Durand et al., 1996; Isaac, 2003; Rumpel et al., 2004; Busetto et al., 2008; Kerchner and Nicoll, 2008). During this period of development, the brain is highly prone to seizures and epileptogenesis (Talos et al., 2006). Consistent with other reports, we show a considerable reservoir of silent synapses in the normal immature rat (Isaac et al., 1997). However, following hypoxia-induced seizures at P10, silent synapses are greatly reduced compared with age-matched controls. The evidence for unsilencing comes from (1) the post-HS increase in AMPAR to NMDAR-mediated sEPSC frequency ratio and an increase in spontaneous and miniature EPSC frequency in CA1 pyramidal neurons (Palmer et al., 2004; Rakhade et al., 2008), (2) the decrease in failure rate difference of eEPSCs at -60 and $+40 \mathrm{mV}$, and (3) a greater synaptic overlap of markers for NMDAR and AMPAR subunits in the area of the dendritic fields of CA1 pyramidal neurons. Interestingly, animals exposed to hypoxia without seizures failed to show any change in silent synapses, suggesting that seizure activity is critical to decreasing silent synapse following HS.

The current data build upon our previous work showing early HS-induced posttranslational modification of AMPARs consistent with trafficking into synapses (Rakhade et al., 2008). The diminution of silent synapses observed following HS occurs shortly after the changes in posttranslational phosphorylation of the GluA1 subunit of the AMPAR at Ser831 and Ser845 (Rakhade et al., 2008), which have been implicated in modifying kinetics and increasing insertion into synapses (Hayashi and Huganir, 2004; Boehm et al., 2006; Shepherd and Huganir, 2007). This is consistent with the post-HS increase in AMPAR number per synapse shown here by IHC and peak-scaled nonstationary analysis.

Mokin et al. (2007) observed similar findings of increased triple-labeling in the setting of synaptic plasticity, with silent synapses decreasing after in vitro classical conditioning. In our experiments, the percentage of NR1-only synapses decreased by approximately half, which was less dramatic than the decrease measured with electrophysiology. This is likely because to accurately compare control and post-HS, we analyzed puncta in a small area of CA1, which may not be representative of the entire dendritic tree. Therefore, it is imperative to interpret these data in light of the electrophysiology.

The changes described here are likely to be due largely to a postsynaptic mechanism. First, we see a large postsynaptic change in synaptic GluA1 expression after seizures. Also, we show evidence that there is no coincident change in paired-pulse responses in slices from post-HS rats compared with controls. Finally, neurons in control slices had the expected larger coefficient AMPAR-eEPSC variances with smaller NMDAR-eEPSCs compared with post-HS, consistent with a postsynaptic change after HS. 


\section{Neonatal seizures can impair subsequent and adult LTP}

Neonatal seizures, including HS, can impair later-life learning and synaptic plasticity (Yang et al., 2004; Holmes and Ben-Ari, 2007). Here, we show that at least one consequence of HS is subacute attenuation in LTP $48-72 \mathrm{~h}$ after HS, in a developmental window in which enhanced synaptic plasticity is critical to normal brain development (Dumas, 2005). Seizures induce a rapid and transient increase in phosphorylation of GluA1 at Ser831/845 (Rakhade et al., 2008). Acute GluA1 phosphorylation at these sites is linked to a decrease in LTP induction threshold (Makino et al., 2011), and indeed we have previously reported an enhancement of LTP immediately after seizure onset at this early time point (Jensen et al., 1998). The enhanced LTP at P10 is likely due to a persistent pool of available silent synapses in the presence of an increased population of phosphorylated GluA1 subunits: the latter has been linked to enhanced LTP (Makino et al., 2011). However, by $48-72 \mathrm{~h}$, GluAl phosphorylation and activity of the responsible kinases return to baseline (Rakhade et al., 2008), coincident with the decrease in silent synapse fraction reported here. Hence, the silent synapse depletion provides a likely mechanism contributing to the LTP attenuation observed here at $48-72 \mathrm{~h}$ after seizure (Kerchner and Nicoll, 2008).

Because LTP is thought to be integral for learning and memory (Whitlock et al., 2006), early synaptic plasticity disruption can profoundly affect cognitive development. Following various models of induced neonatal seizures, rodents show acute and long-term deficits in spatial learning and memory (Huang et al., 2002; Cornejo et al., 2008; Karnam et al., 2009). Indeed in this model, seizures in early life appeared to disrupt synaptic plasticity both subacutely and also long term. LTP was impaired in adult rats with prior HS, suggesting that there are permanent modifications in hippocampal network plasticity. These results are consistent with prior reports in this HS model of impaired learning in adults when tested in vivo (Mikati et al., 2005).

In humans, a variety of cognitive impairments, including memory deficits and intellectual disability, often present following seizures (Dodrill, 2002; Vingerhoets, 2006; Hoppe et al., 2007; Carreño et al., 2008; Patrikelis et al., 2009). A number of reports also show a correlation between epileptiform EEG abnormalities and cognitive impairment, and that seizure control may improve intellectual function in this setting (Spence and Schneider, 2009; Tuchman et al., 2010). This raises the question of whether early seizure control may prevent these alterations in cognitive dysfunction. An emerging body of literature shows that seizures are comorbid with several pediatric neurological disorders with aberrant synaptic plasticity, including autism spectrum disorders (Bassell and Warren, 2008; Jensen, 2009; Hoeffer and Klann, 2010). For example, in mouse models of Rett syndrome (Asaka et al., 2006; Moretti et al., 2006) and tuberous sclerosis (von der Brelie et al., 2006), hippocampal LTP is impaired in a manner similar to that reported here following HS: interestingly these animal models also exhibit spontaneous seizures as part of their phenotype. Our present results raise the possibility that early life seizures have a causative role, with postseizure alterations in synaptic plasticity providing a possible underlying mechanism for cognitive and memory deficits in epilepsy patients.

\section{Postseizure treatment with the AMPAR antagonist NBQX is protective against alterations in silent synapses and LTP}

Our data suggest that neonatal HS can occlude LTP by co-opting mechanisms normally subserving activity-dependent activation of silent synapses, specifically by diminishing the pool of available silent synapses. We have previously reported that AMPAR acti- vation was required following neonatal $\mathrm{HS}$ to induce kinasemediated GluA1 Ser831 and Ser845 phosphorylation, and that in vivo postseizure treatment within $48 \mathrm{~h}$ with AMPAR antagonists NBQX, topiramate, or GYKI-53773, prevented these alterations as well as the HS-enhanced AMPAR EPSCs (Rakhade et al., 2008). These studies show that ongoing AMPAR activation appears critical for seizure-induced posttranslational changes in GluA1. Importantly, in this early period of development, we and others have shown that many AMPARs in hippocampus and cortex are lacking the GluA2 subunit, and are hence $\mathrm{Ca}^{2+}$ permeable and thus can impact activity-dependent signaling cascades involved in synaptic plasticity (Rakhade and Jensen, 2009). Hence, we reasoned that if the HS-induced increase in AMPAR function contributed to the silent synapse reduction, treatment with AMPAR antagonist NBQX might prevent this loss and preserve LTP. Indeed, despite animals having the same number of seizures as controls, post-HS treatment in vivo with NBQX resulted in preservation of silent synapses at levels similar to that of naive, age-matched controls, while NBQX treatment in controls did not cause a significant (only trending) increase in silent synapses. Moreover, slices removed from NBQX-treated rats showed similar capacity for LTP as controls, suggesting that treatment preserved normal network functioning. Although the mechanism of NBQX protection is likely to be multifactorial, our data suggest that it may involve regulation of silent synapses through initiating AMPARs internalization (Lin et al., 2000). Of clinical relevance, these data also suggest that some seizure-induced alterations in network plasticity may be preventable by immediate postseizure treatment timed during the first $24-48 \mathrm{~h}$, when activity-dependent kinases and AMPAR subunit phosphorylation peak (Rakhade et al., 2008).

Dysregulation of the balance between silent and active synapses by seizures in early life also has long-lasting effects on hippocampal plasticity. Here, we report that there are persistent impairments of LTP into adulthood. Importantly, the early postseizure NBQX posttreatment paradigm protected against the alterations into adulthood. The efficacy of NBQX here serves as proof-of-concept that early triggers for dysregulation of silent synapse expression contributes to long-term changes in neuronal networks. Learning and memory impairments are common long-term consequences of epilepsy, and these data suggest that it may be possible to prevent altered synaptic plasticity both acutely and long term using rapid postseizure treatment with AMPAR antagonists. Currently, there are drugs with AMPAR antagonist actions that are either FDA-approved for other indications, such as topiramate, or under investigation in clinical trials, such as GYKI-53773. Both NBQX and topiramate show potential safety, as they do not induce apoptosis in the developing brain, in contrast to other anticonvulsants such as phenobarbital, valproate, and phenytoin (Bittigau et al., 2002). The protective effects seen here, clinical availability, and potential safety of AMPAR antagonists suggests they may represent a strategy for prophylaxis against the effects of HS-induced synaptic dysplasticity in the neonate.

In conclusion, this work provides direct evidence that neonatal HS can change synapse function and have profound and longlasting effects on subsequent neuronal network excitability and synaptic plasticity, which can be prevented by rapid postseizure treatment with AMPAR antagonists. These changes could in part underlie cognitive and memory deficits seen following early life seizures, and point to AMPARs as therapeutic targets for early intervention in epilepsy to target both epileptogenesis and cognitive comorbidities. 


\section{References}

Asaka Y, Jugloff DG, Zhang L, Eubanks JH, Fitzsimonds RM (2006) Hippocampal synaptic plasticity is impaired in the Mecp2-null mouse model of Rett syndrome. Neurobiol Dis 21:217-227.

Bassell GJ, Warren ST (2008) Fragile X syndrome: loss of local mRNA regulation alters synaptic development and function. Neuron 60:201-214.

Benke TA, Lüthi A, Isaac JT, Collingridge GL (1998) Modulation of AMPA receptor unitary conductance by synaptic activity. Nature 393:793-797.

Berg AT (2011) Epilepsy, cognition, and behavior: the clinical picture. Epilepsia 52 [Suppl 1]:7-12.

Bittigau P, Sifringer M, Genz K, Reith E, Pospischil D, Govindarajalu S, Dzietko M, Pesditschek S, Mai I, Dikranian K, Olney JW, Ikonomidou C (2002) Antiepileptic drugs and apoptotic neurodegeneration in the developing brain. Proc Natl Acad Sci U S A 99:15089-15094.

Boehm J, Kang MG, Johnson RC, Esteban J, Huganir RL, Malinow R (2006) Synaptic incorporation of AMPA receptors during LTP is controlled by a PKC phosphorylation site on GluR1. Neuron 51:213-225.

Busetto G, Higley MJ, Sabatini BL (2008) Developmental presence and disappearance of postsynaptically silent synapses on dendritic spines of rat layer 2/3 pyramidal neurons. J Physiol 586:1519-1527.

Carreño M, Donaire A, Sánchez-Carpintero R (2008) Cognitive disorders associated with epilepsy: diagnosis and treatment. Neurologist 14:S26-34.

Cornejo BJ, Mesches MH, Benke TA (2008) A single early-life seizure impairs short-term memory but does not alter spatial learning, recognition memory, or anxiety. Epilepsy Behav 13:585-592.

Crump FT, Dillman KS, Craig AM (2001) cAMP-dependent protein kinase mediates activity-regulated synaptic targeting of NMDA receptors. J Neurosci 21:5079-5088.

Dodrill CB (2002) Progressive cognitive decline in adolescents and adults with epilepsy. Prog Brain Res 135:399-407.

Dumas TC (2005) Late postnatal maturation of excitatory synaptic transmission permits adult-like expression of hippocampal-dependent behaviors. Hippocampus 15:562-578.

Durand GM, Kovalchuk Y, Konnerth A (1996) Long-term potentiation and functional synapse induction in developing hippocampus. Nature 381:71-75.

Esteban JA, Shi SH, Wilson C, Nuriya M, Huganir RL, Malinow R (2003) PKA phosphorylation of AMPA receptor subunits controls synaptic trafficking underlying plasticity. Nat Neurosci 6:136-143.

Hayashi T, Huganir RL (2004) Tyrosine phosphorylation and regulation of the AMPA receptor by SRC family tyrosine kinases. J Neurosci 24:6152-6160.

Hoeffer CA, Klann E (2010) mTOR signaling: at the crossroads of plasticity, memory and disease. Trends Neurosci 33:67-75.

Holmes GL, Ben-Ari Y (2007) A single episode of neonatal seizures permanently alters glutamatergic synapses. Ann Neurol 61:379-381.

Hoppe C, Elger CE, Helmstaedter C (2007) Long-term memory impairment in patients with focal epilepsy. Epilepsia 48 [Suppl 9]:26-29.

Huang LT, Yang SN, Liou CW, Hung PL, Lai MC, Wang CL, Wang TJ (2002) Pentylenetetrazol-induced recurrent seizures in rat pups: time course on spatial learning and long-term effects. Epilepsia 43:567-573.

Huttenlocher PR, Wollmann RL (1991) Cellular neuropathology of tuberous sclerosis. Ann NY Acad Sci 615:140-148.

Isaac JT (2003) Postsynaptic silent synapses: evidence and mechanisms. Neuropharmacology 45:450-460.

Isaac JT, Nicoll RA, Malenka RC (1995) Evidence for silent synapses: implications for the expression of LTP. Neuron 15:427-434.

Isaac JT, Hjelmstad GO, Nicoll RA, Malenka RC (1996) Long-term potentiation at single fiber inputs to hippocampal CA1 pyramidal cells. Proc Natl Acad Sci U S A 93:8710-8715.

Isaac JT, Crair MC, Nicoll RA, Malenka RC (1997) Silent synapses during development of thalamocortical inputs. Neuron 18:269-280.

Jensen FE (2009) Introduction-epileptogenic cortical dysplasia: emerging trends in diagnosis, treatment, and pathogenesis. Epilepsia 50 [Suppl 9]:1-2.

Jensen FE, Holmes GL, Lombroso CT, Blume HK, Firkusny IR (1992) Agedependent changes in long-term seizure susceptibility and behavior after hypoxia in rats. Epilepsia 33:971-980.

Jensen FE, Blume H, Alvarado S, Firkusny I, Geary C (1995) NBQX blocks acute and late epileptogenic effects of perinatal hypoxia. Epilepsia 36:966-972.
Jensen FE, Wang C, Stafstrom CE, Liu Z, Geary C, Stevens MC (1998) Acute and chronic increases in excitability in rat hippocampal slices after perinatal hypoxia in vivo. J Neurophysiol 79:73-81.

Karnam HB, Zhao Q, Shatskikh T, Holmes GL (2009) Effect of age on cognitive sequelae following early life seizures in rats. Epilepsy Res 85:221-230.

Kerchner GA, Nicoll RA (2008) Silent synapses and the emergence of a postsynaptic mechanism for LTP. Nat Rev Neurosci 9:813-825.

Koh S, Tibayan FD, Simpson JN, Jensen FE (2004) NBQX or topiramate treatment following perinatal hypoxia-induced seizures prevents later increases in seizure-induced neuronal injury. Epilepsia 45:569-575.

Kullmann DM (1994) Amplitude fluctuations of dual-component EPSCs in hippocampal pyramidal cells: Implications for long-term potentiation. Neuron 12:1111-1120.

Liao D, Hessler NA, Malinow R (1995) Activation of postsynaptically silent synapses during pairing-induced LTP in CA1 region of hippocampal slice. Nature 375:400-404.

Liao D, Scannevin RH, Huganir R (2001) Activation of silent synapses by rapid activity-dependent synaptic recruitment of AMPA receptors. J Neurosci 21:6008-6017.

Lin JW, Ju W, Foster K, Lee SH, Ahmadian G, Wyszynski M, Wang YT, Sheng M (2000) Distinct molecular mechanisms and divergent endocytotic pathways of AMPA receptor internalization. Nat Neurosci 3:1282-1290.

Lisman J, Raghavachari S (2006) A unified model of the presynaptic and postsynaptic changes during LTP at CA1 synapses. Sci STKE 356:re11.

Losi G, Prybylowski K, Fu Z, Luo JH, Vicini S (2002) Silent synapses in developing cerebellar granule neurons. J Neurophysiol 87:1263-1270.

Makino Y, Johnson RC, Yu Y, Takamiya K, Huganir RL (2011) Enhanced synaptic plasticity in mice with phosphomimetic mutation of the GluA1 AMPA receptor. Proc Natl Acad Sci U S A 108:8450-8455.

Malinow R, Malenka RC (2002) AMPA receptor trafficking and synaptic plasticity. Annu Rev Neurosci 25:103-126.

Marie H, Morishita W, Yu X, Calakos N, Malenka RC (2005) Generation of silent synapses by acute in vivo expression of CaMKIV and CREB. Neuron 45:741-752.

Mikati MA, Zeinieh MP, Kurdi RM, Harb SA, El Hokayem JA, Daderian RH, Shamseddine A, Obeid M, Bitar FF, El Sabban M (2005) Long-term effects of acute and of chronic hypoxia on behavior and on hippocampal histology in the developing brain. Brain Res Dev Brain Res 157:98-102.

Mokin M, Keifer J (2006) Quantitative analysis of immunofluorescent punctate staining of synaptically localized proteins using confocal microscopy and stereology. J Neurosci Methods 157:218-224.

Mokin M, Zheng Z, Keifer J (2007) Conversion of silent synapses into the active pool by selective GluR1-3 and GluR4 AMPAR trafficking during in vitro classical conditioning. J Neurophysiol 98:1278-1286.

Moretti P, Levenson JM, Battaglia F, Atkinson R, Teague R, Antalffy B, Armstrong D, Arancio O, Sweatt JD, Zoghbi HY (2006) Learning and memory and synaptic plasticity are impaired in a mouse model of Rett syndrome. J Neurosci 26:319-327.

Nie D, Di Nardo A, Han JM, Baharanyi H, Kramvis I, Huynh T, Dabora S, Codeluppi S, Pandolfi PP, Pasquale EB, Sahin M (2010) Tsc2-Rheb signaling regulates EphA-mediated axon guidance. Nat Neurosci 13:163-172.

Palmer MJ, Isaac JT, Collingridge GL (2004) Multiple, developmentally regulated expression mechanisms of long-term potentiation at CA1 synapses. J Neurosci 24:4903-4911.

Patrikelis P, Angelakis E, Gatzonis S (2009) Neurocognitive and behavioral functioning in frontal lobe epilepsy: a review. Epilepsy Behav 14:19-26.

Petralia RS, Esteban JA, Wang YX, Partridge JG, Zhao HM, Wenthold RJ, Malinow R (1999) Selective acquisition of AMPA receptors over postnatal development suggests a molecular basis for silent synapses. Nat Neurosci 2:31-36.

Rakhade SN, Jensen FE (2009) Epileptogenesis in the immature brain: emerging mechanisms. Nat Rev Neurol 5:380-391.

Rakhade SN, Zhou C, Aujla PK, Fishman R, Sucher NJ, Jensen FE (2008) Early alterations of AMPA receptors mediate synaptic potentiation induced by neonatal seizures. J Neurosci 28:7979-7990.

Rakhade SN, Klein PM, Huynh T, Hilario-Gomez C, Kosaras B, Rotenberg A, Jensen FE (2011) Development of later life spontaneous seizures in a rodent model of hypoxia-induced neonatal seizures. Epilepsia 52:753-765.

Ronen GM, Buckley D, Penney S, Streiner DL (2007) Long-term prognosis 
in children with neonatal seizures: a population-based study. Neurology 69:1816-1822.

Rumpel S, Kattenstroth G, Gottmann K (2004) Silent synapses in the immature visual cortex: layer-specific developmental regulation. J Neurophysiol 91:1097-1101.

Sanchez RM, Koh S, Rio C, Wang C, Lamperti ED, Sharma D, Corfas G, Jensen FE (2001) Decreased glutamate receptor 2 expression and enhanced epileptogenesis in immature rat hippocampus after perinatal hypoxia-induced seizures. J Neurosci 21:8154-8163.

Sanchez RM, Dai W, Levada RE, Lippman JJ, Jensen FE (2005) AMPA/ kainate receptor-mediated downregulation of GABAergic synaptic transmission by calcineurin after seizures in the developing rat brain. J Neurosci 25:3442-3451.

Scantlebury MH, Galanopoulou AS, Chudomelova L, Raffo E, Betancourth D, Moshé SL (2010) A model of symptomatic infantile spasms syndrome. Neurobiol Dis 37:604-612.

Shepherd JD, Huganir RL (2007) The cell biology of synaptic plasticity: AMPA receptor trafficking. Annu Rev Cell Dev Biol 23:613-643.

Silverstein FS, Jensen FE (2007) Neonatal seizures. Ann Neurol 62:112-120.

Spence SJ, Schneider MT (2009) The role of epilepsy and epileptiform EEGs in autism spectrum disorders. Pediatr Res 65:599-606.

Talos DM, Follett PL, Folkerth RD, Fishman RE, Trachtenberg FL, Volpe JJ, Jensen FE (2006) Developmental regualtion of alpha-amino-3-hydroxy-5- methyl-4-isoxazole-proprionic acid receptor subunit expression in forebrain and relationship to regional susceptibility to hypoxic/ischemic injury. II. Human cerebral white matter and cortex. J Comp Neurol 497:61-77.

Thiagarajan TC, Lindskog M, Malgaroli A, Tsien RW (2007) LTP and adaptation to inactivity: overlapping mechanisms and implications for metaplasticity. Neuropharmacology 52:156-175.

Tuchman R, Alessandri M, Cuccaro M (2010) Autism spectrum disorders and epilepsy: moving towards a comprehensive approach to treatment. Brain Dev 32:719-730.

Vingerhoets G (2006) Cognitive effects of seizures. Seizure 15:221-226.

von der Brelie C, Waltereit R, Zhang L, Beck H, Kirschstein T (2006) Impaired synaptic plasticity in a rat model of tuberous sclerosis. Eur J Neurosci 23:686-692.

Whitlock JR, Heynen AJ, Shuler MG, Bear MF (2006) Learning induces long-term potentiation in the hippocampus. Science 313:1093-1097.

Yang SN, Huang CB, Yang CH, Lai MC, Chen WF, Wang CL, Wu CL, Huang LT (2004) Impaired SynGAP expression and long-term spatial learning and memory in hippocampal CA1 area from rats previously exposed to perinatal hypoxia-induced insults: beneficial effects of A68930. Neurosci Lett 371:73-78.

Zhou C, Jensen FE, Sucher NJ (2009) Altered development of glutamatergic synapses in layer V pyramidal neurons in NR3A knockout mice. Mol Cell Neurosci 42:419-426. 